\title{
Phase Inconsistencies and multiple Scattering in SAR Interferometry
}

\author{
Francesco De Zan, Mariantonietta Zonno and Paco López-Dekker
}

\begin{abstract}
With three coherent SAR images it is possible to form three interferograms. In some cases the phases of the three averaged interferograms will be significantly inconsistent and indicate a sort of phase excess or deficit (which we call lack of triangularity or inconsistency). In this paper we illustrate theoretically which models can explain such phenomenon and provide some real-data examples. It is also shown that two or more independent scattering mechanisms are necessary to explain phase inconsistencies. The observation of lack of consistency might be useful to derive information on the target and also as a warning that the scatterer presents a temporal covariance matrix which is not intrinsically real, with consequences for the processing of interferometric stacks.
\end{abstract}

Index Terms-SAR interferometry, closure phase, inconsistency, multiple scattering, skewness

\section{INTRODUCTION}

This paper deals with the issue of lack of consistency in SAR interferometry. To observe this property it is necessary to have three images able to interfere with each other, generating three interferograms. If the interferograms are averaged spatially and the phases are combined in a circular way $\left(\phi_{12}+\phi_{23}+\phi_{31}\right)$, the result might or might not be close to zero. If it is zero (modulo $2 \pi$ ), the three phases are consistent. One interferogram of the three is redudant and could be computed from the other two.

From the pure mathematical point of view there is no need for the three phases to be consistent, but interferometric models often assume consistency, although they do not postulate it explicitly. This fact makes the goal of this paper more difficult, because before showing how and when consistency is violated, it is first necessary to convince the readers why they should expect consistency in the first place. The observation of lack of consistency challenges all simple interpretations of the interferometric phase in terms of pure propagation and require the simultaneous presence of multiple scatterers or multiple scatterer populations.

One must also say that the lack of compensation could simply reflect the presence of statistical noise. In this paper we are going to investigate the non-trivial cases in which inconsistency is not caused by statistical noise, but it has instead a systematic character which reveals some physical cause. In other words, we are interested in inconsistencies at the level of expected values, or inconsistencies that survive a sufficient amount of spacial averaging (multilooking).

The rest of the paper is structured as follows: In Section II we shall introduce the notation and prove that pure propagative

The authors are with the DLR (German Aerospace Center), Oberpfaffenhofen, D-82230 Wessling, Germany (e-mail:francesco.dezan@dlr.de). effects always yield consistent phases. We shall also elaborate on the meaning of consistency for interferometric stacks in terms of real covariance matrices. Section III shall show that two scattering mechanisms are enough to break consistency and give a geometrical representation thanks to a strong analogy with quantum mechanical concepts. In Sections IV and $\mathrm{V}$ we shall analyze various scattering models which foresee consistency violations, supported with real-data examples. In section VI we analyze the case of pure statistical effects. Section VII will deal with the effects on InSAR stack processing and section VIII shall conclude the paper.

\section{Phase CONSISTENCY DEFINITION AND MEANING}

Let us consider a stack of coregistered and interferometrically compatible SAR images. Calling $i_{n}$ the $n^{\text {th }}$ image in the stack, the interferogram between image $n$ and image $k$ will be

$$
I_{n k}=\left|I_{n k}\right| \exp \left(j \phi_{n k}\right)=\overline{i_{n} i_{k}^{*}}
$$

where the asterisk stands for the complex-conjugate and the overline denotes some local spatial averaging. It is possible then to define the phase [1]

$$
\Phi_{n k h}=\phi_{n k}+\phi_{k h}+\phi_{h n},
$$

which is also the phase of the triple product $I_{n k} I_{k h} I_{h n}$. In the field of astronomical imaging this phase is called closure phase [2]. In 1958, Jennison was the first to introduce it [3]. An analogy with a quantum-mechanical invariant will be discussed later in this paper.

Under many circumstances the three phases $\phi_{n k}, \phi_{k h}$, and $\phi_{h n}$ will sum up to zero (modulo $2 \pi$ ), up to some statistical noise. There are however cases in which this compensation will not happen and the lack of consistency points to some underlying physical reason related to the scattering mechanism.

In geometrical terms, the inconsistency of the angles measured along a closed path is connected to the idea of curvature (Figure 1). With the analogy to curvature one could say that phase consistency corresponds to a flat geometry, phase excess or deficit to positive or negative curvatures. We will show that in some cases it is possible to give a precise geometrical meaning to the intuition that we are dealing with a curved space.

It has to be stressed that the application of spatial averaging is necessary to reveal possible deviations from zero (inconsistencies) of the closure phase; in fact, for single pixels, one can trivially show that it is always $\Phi_{n k h} \equiv 0$. On the other hand, for expected values or extensive averaging phase consistency 
is not guaranteed: however most coherence models for SAR interferometry implicitly assume it, so that it is meaningful to investigate their validity.
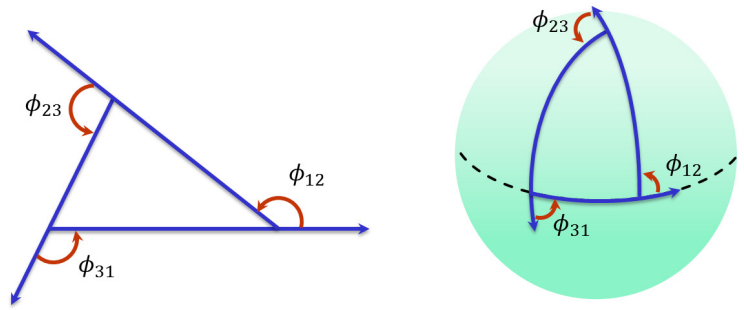

$$
\phi_{12}+\phi_{23}+\phi_{31}=0(2 \pi)
$$$$
\phi_{12}+\phi_{23}+\phi_{31} \neq 0 \quad(2 \pi)
$$

Fig. 1. Classical example of phase consistency for a flat geometry (triangle on a plane, left) and phase inconsistency for a curved surface (triangle on a sphere, right).

\section{A. Pure propagative effects and phase calibration}

It is important to understand which effects do not break the consistency, since their presence is irrelevant: it is not necessary to worry about phase calibration (troposphere, topography, motion) if we are only interested in $\Phi_{n k h}$. In general, all pure delaying effects that affect indepedently each image, like tropospheric delays, are irrelevant for phase consistency.

For example, if the phase of a small area is only affected by a tropospheric delay of $\varphi_{n}^{\text {tropo }}$ (depending on the image index $n$ ), the interferometric phase between two images will see the differential troposheric delay:

$$
\phi_{n k}=\varphi_{n}^{\text {tropo }}-\varphi_{k}^{\text {tropo }}
$$

and $\Phi_{n k h}=0$ since all terms $\varphi_{n}^{\text {tropo }}, \varphi_{k}^{\text {tropo }}, \varphi_{h}^{\text {tropo }}$ will appear twice in the sum (2) with opposite signs.

Similar reasoning can be conducted for phase effects caused by target motion (crustal deformation) and topography in the presence of a normal baseline. In other words: the phase excess or deficit revealed by the three interferograms is invariant under phase rotation of any of the three images. This means that in standard situations we should expect the closure phase to be zero.

\section{B. Interpretation of the interferometric phase for InSAR stacks}

For InSAR stacks the lack of consistency challenges any simple interpretation of the interferometric phase. In particular, when phases are not consistent (intrinsically, i.e. also for large averages, or expected values), it is not immediate to extract a phase vector or phase history from a complex covariance matrix, following an approach like those presented in [4], [5], [6], which aim at transforming a distributed target into an equivalent point target. The phase difference between two images could in general depend on the chosen path, since $\phi_{n k}+\phi_{k h}$ could differ from $\phi_{n h}$ (another way to cast $\Phi_{n k h} \neq 0$ ). It is no longer clear what it means replacing a distributed scatterer by an equivalent persistent one, which has a defined (path-independent) phase history.
The phase inconsistency is equivalent to the impossibility of writing the complex covariance matrix as:

$$
\mathbf{C}=\boldsymbol{\Delta}^{*} \mathbf{C}_{0} \boldsymbol{\Delta},
$$

where $\mathbf{C}_{\mathbf{0}}$ is a real covariance matrix and $\boldsymbol{\Delta}$ is a diagonal matrix with pure phase terms, one phase term per image. This is a fundamental assumption for the covariance-based algorithms in [5], [6], [7] or also [8], [9], which are thus vulnerable to mis-modeling when consistency is not valid. Similar considerations apply to the techniques of the Small Baseline Subset family [10], [11], which are potentially even more affected by inconsistencies, because of the reduced number of interferograms used.

Of course there are many cases in which the consistency hypothesis is satisfied to a sufficient degree, at least for expected values of the covariance matrices or large averages; however, as we are going to show in the following, it is also not so hard to find exceptions at all frequencies of typical SAR operation.

\section{Higher-order combinations}

One could be tempted to extend the definition of $\Phi_{n k h}$ to higher orders, considering four or more images. However there seems to be no additional information in higher-order combinations, since they can be resolved into sums or thirdorder terms. For instance:

$$
\begin{aligned}
\Phi_{1234} & =\phi_{12}+\phi_{23}+\phi_{34}+\phi_{41}= \\
& =\phi_{12}+\phi_{23}+\phi_{31}+\phi_{13}+\phi_{34}+\phi_{41}= \\
& =\Phi_{123}+\Phi_{134} .
\end{aligned}
$$

Indeed not even all $\Phi_{n k h}$ are independent. If there are $N(N-1)(N-2) / 6$ ways of forming triplets out of a stack of $N$ images, the number of independent phase loops must be smaller than $N(N-1) / 2$, i.e., the number of possible interferograms. Actually the number of independent $\Phi_{n k h}$ is $(N-1)(N-2) / 2$, as one can see from writing [2]:

$$
\Phi_{n k h}=\Phi_{0 n k}+\Phi_{0 k h}+\Phi_{0 h n} .
$$

The gap between the number of independent interferometric phases and independent closure phases is $N-1$.

\section{Abstract discussion on the origin of Phase INCONSISTENCIES}

Phase inconsistencies can arise when different scatterer populations with independent phase behaviors interfere with each other. To investigate the origin of phase inconsistencies we study a few simple scenarios in detail.

\section{A. Two (or more) scatterers with changing relative phase}

A possibility to have a phase residual different from zero is to have two scatterers with phases which vary independently from each other in the three images:

$$
\begin{aligned}
& i_{1}=a+b e^{j \varphi_{1}} \\
& i_{2}=a+b e^{j \varphi_{2}} \\
& i_{3}=a+b e^{j \varphi_{3}} .
\end{aligned}
$$


Here, without loss of generality, we have assumed that the phase of the first scatterer does not change between acquisitions. The two scattering mechanisms are modeled as complex random variables ( $a$ and $b$ ). The corresponding interferograms (expected values) are:

$$
\begin{aligned}
& I_{12}=\mathrm{E}\left[|a|^{2}\right]+\mathrm{E}\left[|b|^{2}\right] e^{j\left(\varphi_{1}-\varphi_{2}\right)} \\
& I_{23}=\mathrm{E}\left[|a|^{2}\right]+\mathrm{E}\left[|b|^{2}\right] e^{j\left(\varphi_{2}-\varphi_{3}\right)} \\
& I_{31}=\mathrm{E}\left[|a|^{2}\right]+\mathrm{E}\left[|b|^{2}\right] e^{j\left(\varphi_{3}-\varphi_{1}\right)},
\end{aligned}
$$

assuming incorrelation between the two scattering mechanisms: $\mathrm{E}\left[a b^{*}\right]=0$. According to this model, each interferogram is the sum (indeed the interference) of two more fundamental interferograms. It is possible to show that the resulting residual $\Phi_{123}$ will always different from zero, except in the following cases:

- $\mathrm{E}\left[|a|^{2}\right]=0$ or $\mathrm{E}\left[|b|^{2}\right]=0$, which actually corresponds to one single scattering mechanism;

- $\varphi_{1}=\varphi_{2}$ or $\varphi_{2}=\varphi_{3}$ or $\varphi_{3}=\varphi_{1}$, which corresponds to having two identical images;

- $\mathrm{E}\left[|a|^{2}\right]=\mathrm{E}\left[|b|^{2}\right]$, the only non-trivial case of phase compensation.

An illustration of this abstract model with only phase changes could be a simple across-track tomographic scenario with two layers. The phases $\varphi_{1}, \varphi_{2}, \varphi_{3}$ would then represent the topographic phase of the second scatterer with respect to the first. Apart from the special cases mentioned above, the three different look angles will generate a phase inconsistency in the interferograms. The inconsistency is present automatically in tomographic models, even if it is not normally explicitly discussed.

\section{B. Two (or more) scatterers with changing relative intensity}

In the case that the scatterers change only their relative amplitudes, there is no net phase effect in the expected value and thus no phase excess or deficit. For example one could consider the following scenario:

$$
\begin{aligned}
& i_{1}=a+w_{1} b \\
& i_{2}=a+w_{2} b \\
& i_{3}=a+w_{3} b .
\end{aligned}
$$

with $a, b$ being complex random variables, with $\mathrm{E}\left[a b^{*}\right]=0$, and $w_{n}$ being real and positive weights. It is immediate to verify that in this case there is no interferometric phase in any interferogram and consequently no phase excess $\left(\Phi_{123}=0\right)$. This short analysis precludes pure attenuation effects from explaining interferometric observables. For example, penetration alone cannot be the explanation of interferometric phases as conjectured in [12].

\section{Two (or more) scatterers with changing phase and relative intensity}

This is the combination of the previous two scenarios. The change in the relative intensity of the different scatterers can increase the phase inconsistency effect, provided that a phase effect is anyway present. This is the case of the model presented in [1] in which we attempted to explain interferometric effects of soil moisture variations in bare soils. According to that model, variations in the dielectric permittivity (linked to moisture levels) would produce variations in both phase and amplitudes for scatterers at different depths into the soil.

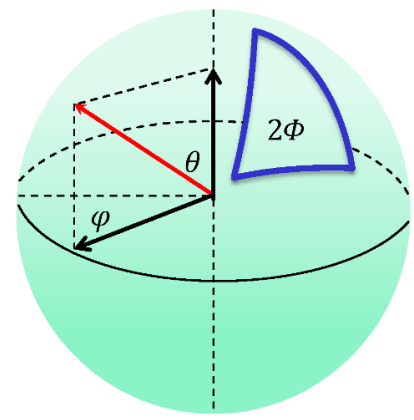

Fig. 2. It is possible to map two orthogonal scattering mechanisms and their combinations as points on Bloch's sphere. Phase inconsistencies for image sequences correspond to areas on the sphere.

In the general case of two scattering mechanisms, each being characterized by a real covariance matrix $\mathbf{C}_{\{\mathbf{a}, \mathbf{b}\}}$, the resulting covariance matrix can be written as:

$$
\mathrm{C}=\Delta_{\mathrm{a}}^{*} \mathrm{C}_{\mathrm{a}} \Delta_{\mathrm{a}}+\Delta_{\mathrm{b}}^{*} \mathrm{C}_{\mathrm{b}} \Delta_{\mathrm{b}}
$$

where $\boldsymbol{\Delta}_{\{\mathbf{a}, \mathbf{b}\}}$ are diagonal matrices with pure phase terms, describing the phase histories of the two scattering mechanisms. This is a straightforward extension of Eq. (4) that is compatible with the presence of inconsistencies.

\section{Analogy with Quantum Mechanics}

Some analogy with physics can be helpful in the study of this interferometric problem. The concept of lack of consistency presented here is very similar to the concept of Bargmann invariant found in quantum physics (see, e.g., [13]). With three quantum states $|1\rangle,|2\rangle$ and $|3\rangle$, the three-point Bargmann invariant is defined, in the standard bra-ket notation, as the quantity

$$
\Delta^{(3)}=\langle 1 \mid 2\rangle\langle 2 \mid 3\rangle\langle 3 \mid 1\rangle \text {. }
$$

Both amplitude and phase are invariant under arbitrary phase rotations of each state.

To make the connection with SAR interferometry one has only to see that SAR image pixels are analogous to quantum states and expected values of interferograms are analogous to inner products of states. Coming to sample averages, for SAR interferometry the average involves pixels in the space dimension, while quantum averages are typically the result of experiments repeated over time.

Recognizing this analogy, several properties of the phase of Bargmann's invariant $\Delta^{(3)}$ can be shown to apply also to $\Phi_{n k h}$, like Eq. (5) or the invariance under phase calibration, which corresponds to rotating a quantum state by a phase factor. 
The particular case of two independent scattering mechanisms (Eq. (9)) contributing to the images can be well represented using Bloch's sphere (Fig. 2), which is used in quantum mechanics to illustrate the quantum bit. We map the two independent mechanisms $a$ and $b$ at two opposite poles of the sphere (North and South pole). Each point will be identified with the usual spherical coordinates $(\theta, \varphi)$ and each image will then be a linear combination of the two chosen independent mechanisms in the following way:

$$
i_{n}=\cos \left(\frac{\theta_{n}}{2}\right) \cdot a+\sin \left(\frac{\theta_{n}}{2}\right) e^{j \varphi_{n}} \cdot b,
$$

with different relative weighting $\left(\theta_{n}\right)$ and relative phase $\left(\varphi_{n}\right)$. Without loss of generality, the two scattering mechanisms are assumed to have the same normalized intensity: $\mathrm{E}\left[|a|^{2}\right]=$ $\mathrm{E}\left[|b|^{2}\right]=1$. In this way, every point on the sphere represents an image, with its unique combination of the scattering mechanisms.

The phase excess $\Phi$ from three images $i_{1}, i_{2}$ and $i_{3}$ is half the area of the spherical triangle having the three images as vertices. Examining Bloch's sphere it is immediately clear that some variations do not produce phase inconsistencies. If the intensities are equal $(\theta=\pi / 2)$, the motion will be along the equator; if the phase $\varphi$ stays constant, the motion will be along a meridian. In both cases the triangles degenerate and their area is zero. Unfortunately it is not possible to identify interferometric coherences as scalar products of the points on the sphere.

\section{VOLUME SCATTERING \& PERPENDICULAR BASELINE}

Volume scattering is probably the simplest case to study the presence and meaning of phase inconsistencies. Tomographic reconstruction from multibaseline SAR acquisitions is a wellunderstood problem, and has been applied to a variety of scenarios. In the following, we show that there is an interpretation of the phase excess in terms of scattering profile moments.

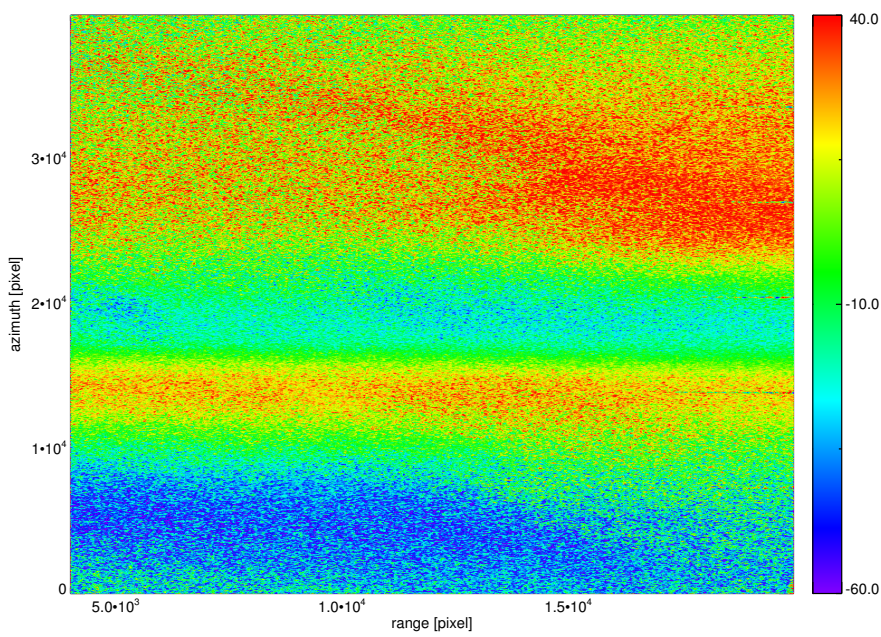

Fig. 3. Phase inconsistency observed over Ronne's ice shelf (Antarctica) acquired by TerraSAR-X mission. The color scale is in degrees. Acquisition dates: 2012-03-04, 2012-03-09, 2012-03-10.
A. Relations between scattering profile moments and the interferometric phase

Let us characterize a volumetric scatterer with a real-valued vertical scattering profile function $f(z)$. In a SAR tomographic setting, we will have different acquisitions taken at slightly different incidence angles, providing some resolution in the elevation direction. Each pair of acquisitions is associated with a vertical (differential) wavenumber $k$, which describes the variation of the differential phase with the height coordinate.

We are going to derive some interesting relations between the vertical scattering profile and the behavior of interferometric phase and coherence. Hensley presented a similar derivation in [14]. The interferogram $I(k)$ as a function of the vertical wavenumber $k$ is the Fourier transform of the profile and has the characteristics of an autocorrelation

$$
I(k)=\int f(z) e^{j k z} d z,
$$

because the profile is real and positive (spectrum). Let us assume that the interferogram and the profile are normalized so that $I(0)=\int f(z) d z=1$. This is equivalent to working with coherences and $f(z)$ is like a probability distribution.

Separating now the phase and amplitude components of the interferogram

$$
I(k)=A(k) e^{j \phi(k)}
$$

and equating the derivatives of Eq. (13) and (14) one can obtain the following interesting relations

$$
\begin{aligned}
\phi^{\prime}(0) & =\mathrm{E}[z]=\mu_{z} \\
A^{\prime \prime}(0) & =-\mathrm{E}\left[\left(z-\mu_{z}\right)^{2}\right] \\
\phi^{\prime \prime \prime}(0) & =-\mathrm{E}\left[\left(z-\mu_{z}\right)^{3}\right] \\
A^{I V}(0) & =\mathrm{E}\left[\left(z-\mu_{z}\right)^{4}\right],
\end{aligned}
$$

which tell us that the derivatives in zero are related to the central moments of the profile (spectrum). In $k=0$ all even derivatives of $\phi(k)$ and all odd derivatives of $A(k)$ are equal to zero, because of the Hermitian symmetry of $I(k)$.

In particular one can see that for $k \approx 0$

$$
\phi(k) \approx \phi^{\prime}(0) k=\mu_{z} k,
$$

which confirms that for small baselines the interferometric phase represents the mean of the vertical scattering profile. To obtain a useful approximation for the closure phase $\Phi_{123}$ it is necessary to expand up to third order in $k$, since $\phi^{\prime \prime}(0)=0$ :

$$
\begin{aligned}
\Phi_{123} & =\phi_{12}+\phi_{23}+\phi_{31} \\
& \approx \phi^{\prime}(0)\left(k_{12}+k_{23}+k_{31}\right)+\frac{1}{6} \phi^{\prime \prime \prime}(0)\left[k_{12}^{3}+k_{23}^{3}+k_{31}^{3}\right] \\
& =-\frac{1}{2} \mathrm{E}\left[\left(z-\mu_{z}\right)^{3}\right] k_{12} k_{23} k_{31} .
\end{aligned}
$$

This result shows how the phase excess is directly dependent on the profile skewness $\mathrm{E}\left[\left(z-\mu_{z}\right)^{3}\right]$, at least for small baselines. To derive Eq. (20) we have exploited the trivial consistency of the vertical wavenumbers: $k_{12}+k_{23}+k_{31}=0$.

It is perhaps worth saying that, although this interpretation of the closure phase in terms of profile skewness might be useful for tomographic applications, we do not believe that existing tomographic algorithms (backprojection, Capon, 


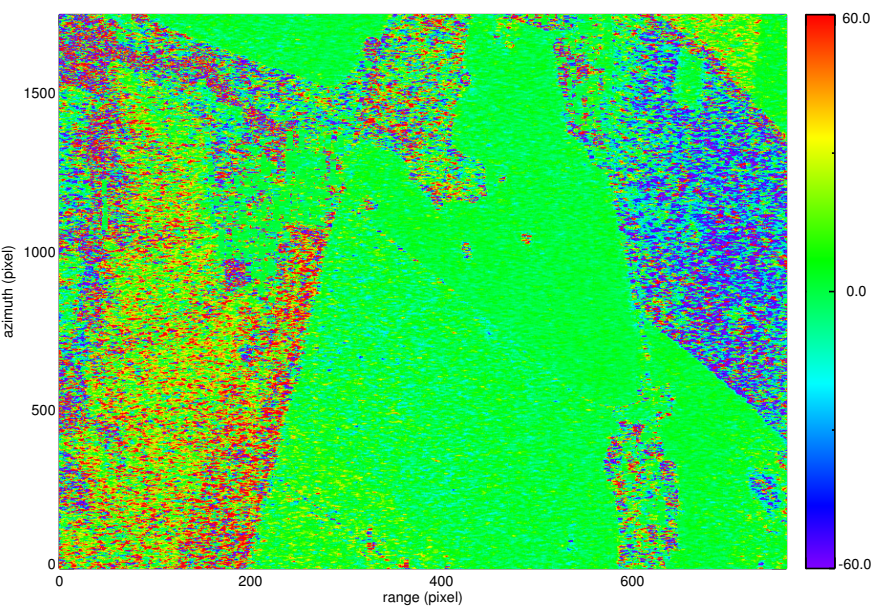

Fig. 4. Closure phase observed over agricultural fields (E-SAR, L-band). The color scale is in degrees. Acquisition dates: 2006-05-16, 2006-06-13, 2006-06-21. Heights of ambiguity: $1087 \mathrm{~m}, 81 \mathrm{~m},-75 \mathrm{~m}$

compressive sensing, model based, etc.) should treat closure phases explicitly, since they already implicitly deal with them.

\section{B. Crossing-orbit experiment data}

Figure 3 provides a real data illustration of lack of triangularity caused by volumetric scattering. With the three acquisitions of a TerraSAR-X crossing-orbit experiment the resulting closure phase $\Phi_{123}$ differs from zero by several dozens of degrees. The two slaves are separated from the master by 1 and 5 days. The azimuth variations are mainly caused by the variation of the baselines within the scene, typical of the crossing orbit geometry. More details on the crossing-orbit experiment can be found in [15].

Since, in this unusual geometry, the effective baselines vary strongly and linearly with azimuth, the term $k_{12} k_{23} k_{31}$ describes a cubic with three zero crossings, which can be clearly seen in the azimuth direction in Figure 3 as horizontal, green stripes.

\section{PRopagation IN A VARIABLE DIELECTRIC}

\section{A. Soil moisture variations}

Another reason that could give rise to systematically imperfect compensation is a variation of soil moisture according to the model presented in [1]. There it is assumed that the scattered energy comes from targets at different depths, with propagation phases which depend both on the moisture state and the depth. The interferogram $I_{1,2}$ between two acquisitions will be given by the integral:

$$
I\left(k_{1}, k_{2}\right)=\int_{0}^{\infty} f(z) e^{-j k_{1} z}\left(e^{-j k_{2} z}\right)^{*} d z
$$

where the $k$ 's are the moisture-dependent vertical wavenumbers in the soil and $f(z)$ is a scattering profile. Such a model is rather general and predicts inconsistencies for different forms of $f(z)$. This is the case, for instance, for $f(z)=1$ since the exponential attenuation in the exponent provides the necessary skewness or asymmetry. It is also conceivable to have concentrated scatterers in $f(z)$, like Dirac's deltas, corresponding to

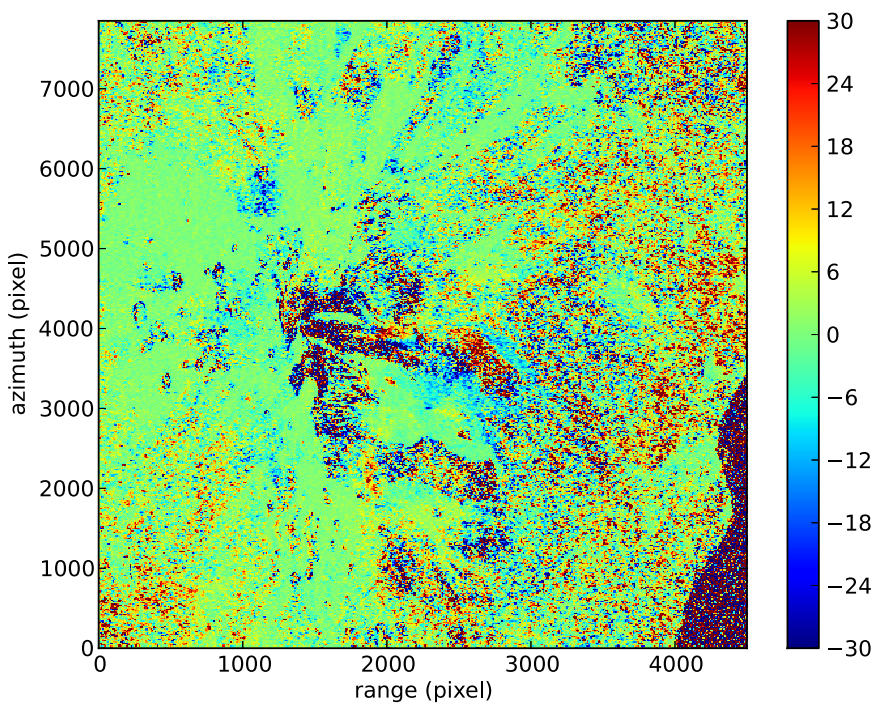

Fig. 5. Closure phase observed over Mt. Etna (Italy) acquired by PALSAR The color scale is in degrees. Acquisition dates: 2007-06-14, 2007-07-30, 2007-09-14. Heights of ambiguity: $-112 \mathrm{~m},-419 \mathrm{~m},+88 \mathrm{~m}$.

strong reflections either at the air-soil interface or at some deeper permittivity discontinuity: in this case the model will resemble closely to (8). At the moment it is not clear which model for $f(z)$ will best describe the observations in a general case: to answer this question it will be necessary to examine extensively real data.

With a continuous $f(z)$, the resulting effect could be described as volumetric but in this case the volume consists in just a few centimeters of soil and the moisture variation plays the role of normal baseline (by controlling the vertical wavenumber). There are indeed attempts to conduct tomographic reconstructions in small depths as in [16]. Although the mathematical expressions are different, the complex coherences modeled in [1] belong almost perfectly to Dall's circle for infinite volumes described in [17]. The two models, albeit different, share a very similar structure.

For an example of inconsistencies observed over agricultural fields we show in Figure 4 the closure phase derived from three images acquired by DLR's E-SAR L-band radar [18]. More details can be found in [1].

Figure 5 shows the lack of triangularity in three L-band ALOS-PALSAR images acquired over Mt. Etna. For the area toward the montain summit, since there are no trees, the moisture variation hypothesis seems the most likely to explain the phase excess. Other examples over the same area show very different patterns as displayed in Fig. 6. Since the mountain top is usually covered with snow during winters, it is possible that snow melt produces moisture variations appearing in the closure phase.

\section{B. Vegetation water content variations}

In some observations the phase inconsistency seems to be related to the presence of forest, even though the normal baselines are not large enough to justify pure volumetric effects. For these cases a possible explanation is a variation 

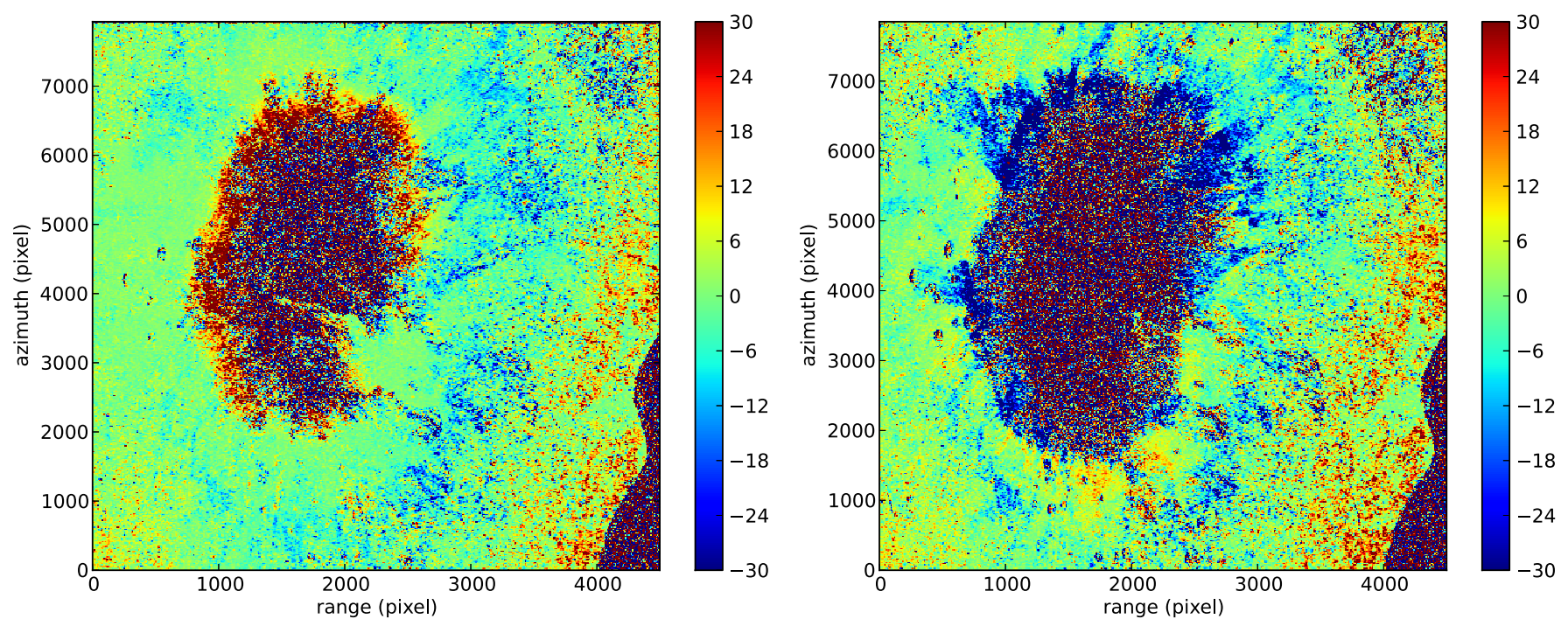

Fig. 6. Closure phase observed over Mt. Etna (Italy) acquired by PALSAR. The color scale is in degrees. Left: Acquisition dates: 2007-12-15, 2008-01-30, 2008-03-16. Heights of ambiguity: +150 m, -349 m, -103 m. Right: Acquisition dates: 2009-12-20, 2010-02-04, 2010-03-22. Heights of ambiguity: -106 m, $-161 \mathrm{~m},+63 \mathrm{~m}$.
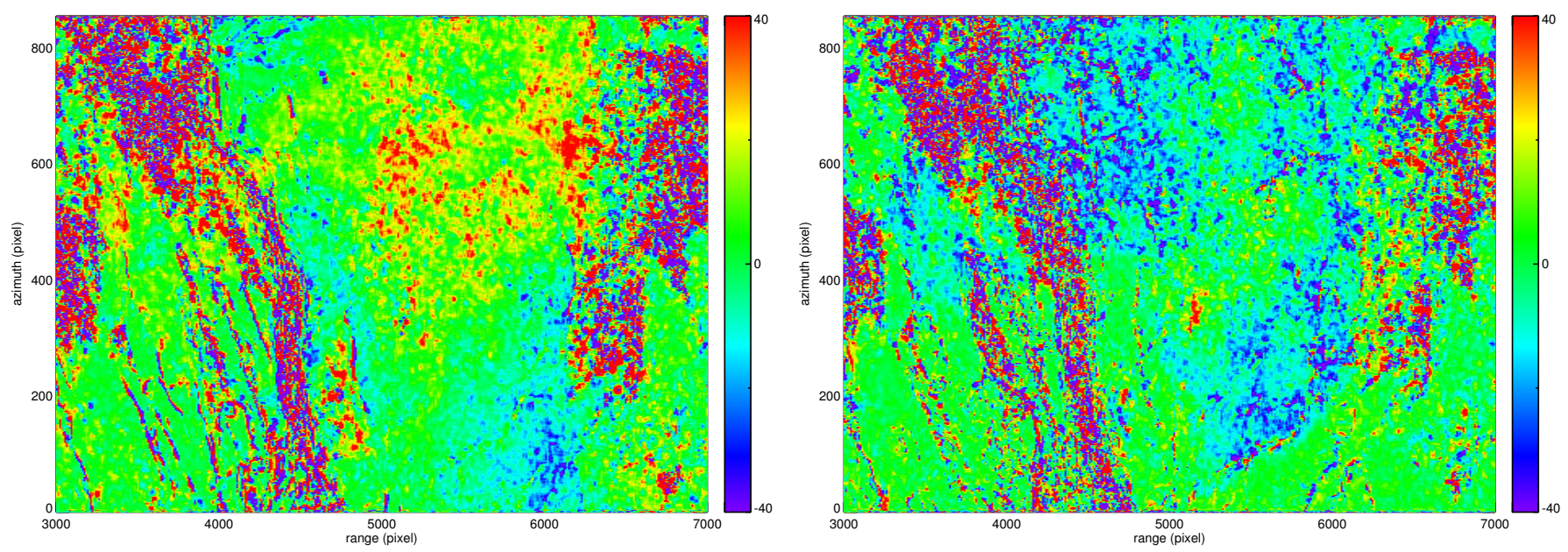

Fig. 7. Closure phase observed over Afar triangle (East Africa) acquired by TerraSAR-X. The color scale is in degrees. Left: Acquisition dates: 2012-07-03, 2012-08-16, 2012-09-07. Heights of ambiguity: -48 m, +15 m, -22 m. Right: Acquisition dates: 2012-06-11, 2012-06-22, 2012-07-25. Heights of ambiguity: $+8 \mathrm{~m},-121.7 \mathrm{~m},-8.6 \mathrm{~m}$.

of water content in the trees themselves and consequently a variation of their dielectric permittivity at different heights.

This hypothesis finds support in the analysis of the TropiScat experiment[19] (ESA, 2011-2012), which acquired almost continuously radar data over the tropical forest from a tower in French Guiana.

The advantage of the TropiScat experiment is that it provides a calibrated phase, therefore coupling effects between coherence magnitude and phase are apparent. Indeed many of the coherences computed with data acquired within 24 hours describe curves in the complex plane, like the one presented in Figure 9 (left) for a particular day. Every point is the complex coherence between two acquisitions in the same day: there are $96 \times 95 / 2=4560$ possible pairs, starting from 96 acquisitions in one day (one every 15 minutes). These clouds remind Eq. (8), with one scatterer moving and the other not. For sure they reveal that behind temporal decorrelation there can sometimes be a coherent mechanism.

In this case the normal baseline is zero and the physical variable driving the phase change could be the dielectric permittivity of sapwood, which is known to vary diurnally with water content and fluid chemistry [20] and directly affects the propagation of electromagnetic waves inside the trees.

Since the TropiScat experiment has also some tomographic capability, it is then possible to separate the contributions from different layers and examine their phases separately. Figure 9 (right) shows the interferometric phase at different heights, with the midnight acquisition as interferometric reference. Although the separation of the different layers cannot be perfect, because of geometrical limitations to the vertical resolution, one layer presents a phase excursion much larger than the others. This layer is around $20 \mathrm{~m}$ above the base of the tower, but because of topography, it corresponds to a variable height of 20-40 $\mathrm{m}$ from the forest bottom. During nighttime the 

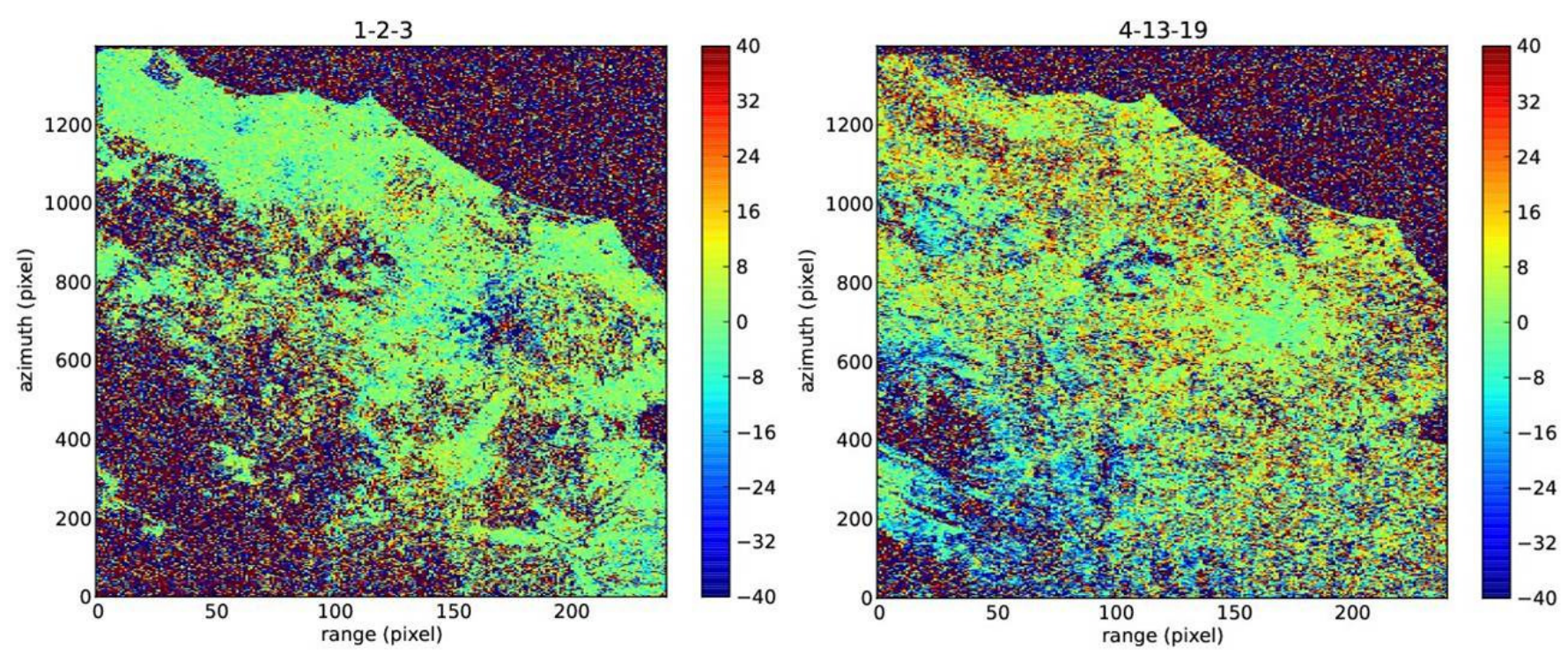

Fig. 8. Closure phase observed over Rome (Italy) acquired by ERS-1. The color scale is in degrees. Left: Acquisition dates: 1993-12-26, 1994-12-29, 1994-01-01. Heights of ambiguity: 53 m, 60 m, -27 m. Right: Acquisition dates: 1994-01-07, 1994-02-24, 1994-03-07. Heights of ambiguity: -698 m, -165 $\mathrm{m},+217 \mathrm{~m}$.

phase shows clear linear trends of up to 40 degrees (negative). These are hard to explain as actual range displacements $(3.3$ $\mathrm{cm}$, moving away from the sensor) but they could be explained with just a few millimeters $(3.7 \mathrm{~mm})$ of water accumulated in the sapwood, using for water a relative permittivity of 80 .

\section{Observations at $C$ and $X$-band}

It is possible to find examples of phase inconsistencies also at higher frequencies, like $\mathrm{C}$ and $\mathrm{X}$-band, although they seem to be less frequent or more difficult to find. Inconsistencies were observed in a desertic area called "the Afar triangle" in East Africa with TerraSAR-X images (Figure 7). Given the small heights of ambiguity involved, it is not clear whether a standard volumetric mechanism is at work or not. Considering that the acquisitions belong to morning passes, and that the patterns look different in the two cases, the moisture hypothesis has some credibility.

Figure 8 presents results obtained over Rome and its surroundings with ERS-1 data from the Ice Phase (3-day repeat). In the first image the relatively small heights of ambiguity point to volumetric effects. The city of Rome appears with mainly blue colors in the right part of the image. In the second image the city area appears in green, which means that the phase is consistent, and indeed the ambiguity heights are all rather large. However forested areas appear to systematically deviate from zero, especially in the bottom-left quadrant. In this case the moisture hypothesis seems necessary to explain the observations.

\section{StATISTICAL EFFECTS}

A final example of lack of phase consistency, not particularly interesting from the perspective of this paper, is connected to the natural statistical variation of sample covariance matrices with respect to ideal covariances. This component is enough to break the perfect triangularity of an ideal realvalued coherency matrix. Indeed one can see the phase-linking algorithm [5], [6] as a tool to restore consistency in the phases of an interferometric stack "corrupted" by statistical (speckle) noise.

The phase mismatch associated with statistical noise will be different (independent) for each averaging window and therefore no systematic bias is expected. This fact allows distinguishing interesting mismatches from trivial ones: if the excess phase is spacially correlated, it can not be a statistical effect. The magnitude of the mismatch will also be an interesting indication, since it will not be reduced by multilooking as regular statistical noise. One reviewer has very rightly suggested that we include in this paper the expression for the closure phase noise. This expression enables the construction of statistical tests to reject the null hypothesis of zero closure phase (expected values). We will thus assume that $\mathrm{E}\left[\Phi_{123}\right]=0$ (consistency in expected value), so that the expression for the variance of $\Phi_{123}$ reduces to the expected value of $\Phi_{123}^{2}$ :

$$
\begin{aligned}
\operatorname{Var}\left[\Phi_{123}\right]= & \mathrm{E}\left[\Phi_{123}^{2}\right]=\mathrm{E}\left[\left(\phi_{12}+\phi_{23}+\phi_{31}\right)^{2}\right] \\
\simeq & \frac{1}{2 N \gamma_{12}^{2} \gamma_{23}^{2} \gamma_{31}^{2}}\left[3 \gamma_{12}^{2} \gamma_{23}^{2} \gamma_{31}^{2}\right. \\
& +\gamma_{12}^{2} \gamma_{23}^{2}+\gamma_{23}^{2} \gamma_{31}^{2}+\gamma_{31}^{2} \gamma_{12}^{2} \\
& \left.-2 \gamma_{12} \gamma_{23} \gamma_{31}\left(\gamma_{12}^{2}+\gamma_{23}^{2}+\gamma_{31}^{2}\right)\right],
\end{aligned}
$$

with $\gamma_{n k}$ being the coherence magnitudes and $N$ the number of independent samples. To derive (23) we have used

$$
\mathrm{E}\left[\phi_{n k} \phi_{n h}\right] \simeq \frac{\gamma_{k h}-\gamma_{n k} \gamma_{n h}}{2 N \gamma_{n k} \gamma_{n h}}
$$

and its reduction, for $i_{k} \equiv i_{h}$, to the well-known Cramér-Rao bound for interferometric phases, under the usual hypothesis of Gaussian speckle. A further generalization of (24) is

$$
\mathrm{E}\left[\phi_{n k} \phi_{h l}\right] \simeq \frac{\gamma_{n l} \gamma_{k h}-\gamma_{n k} \gamma_{h l}}{2 N \gamma_{n k} \gamma_{h l}}
$$



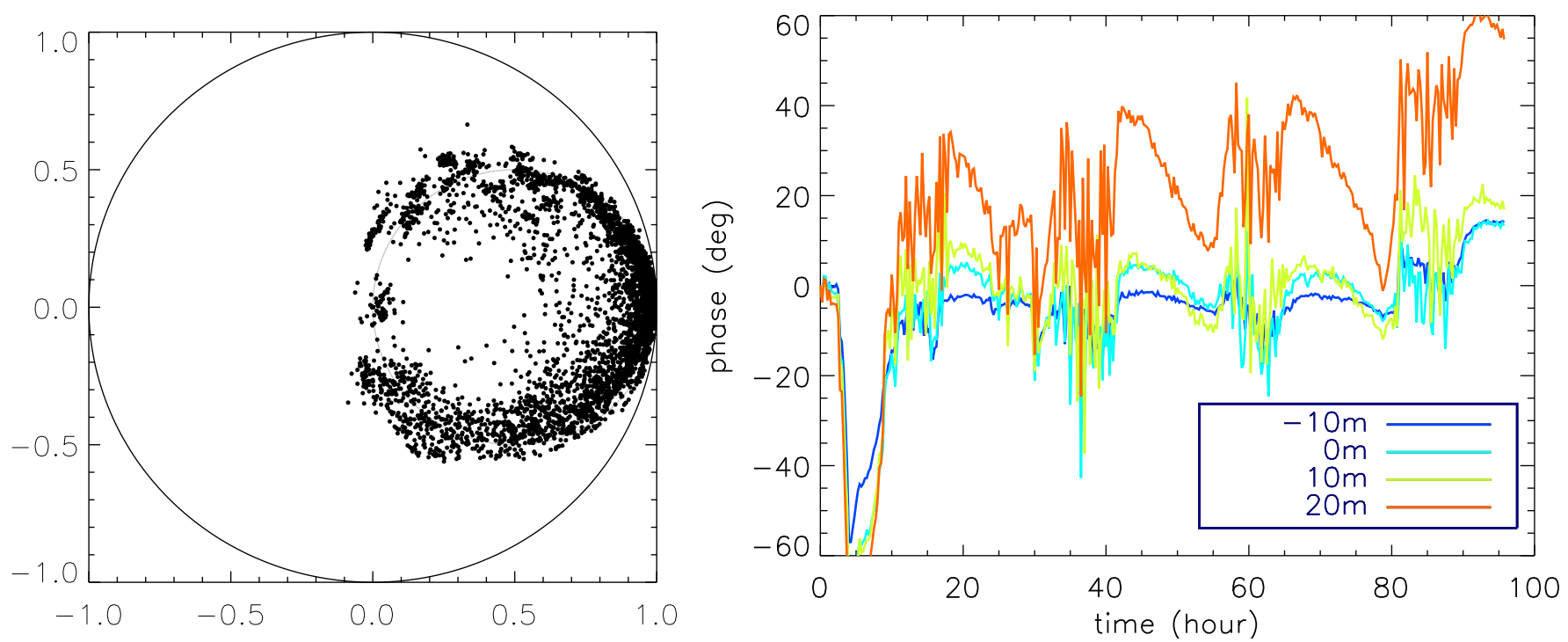

Fig. 9. Left: One example of the complex coherences for the HH channel of the TropiScat experiment (day 2011-12-16, 400-600MHz. Right: Interferometric phase evolution for the $\mathrm{HH}$ channel of the TropiScat experiment after separating tomographically the contributions at different heights (from 2011-12-15H00:00 to $2011-12-18 \mathrm{H} 23: 45,400-600 \mathrm{MHz})$ )

and can also be found in [9], though in a slightly different form. Note that in evaluating (22) it is necessary to account for the cross-terms since the interferometric phases are correlated. In other words, in general, it is:

$$
\mathrm{E}\left[\left(\phi_{12}+\phi_{23}+\phi_{31}\right)^{2}\right] \neq \mathrm{E}\left[\phi_{12}^{2}\right]+\mathrm{E}\left[\phi_{23}^{2}\right]+\mathrm{E}\left[\phi_{31}^{2}\right] .
$$

\section{INTERFEROMETRIC STACK PROCESSING}

It is not straightforward to assess the impact of phase inconsistencies on current algorithms for interferometric stack processing. Apart from the layover case, which a number of algorithms for InSAR stacks treat properly (for instance [21], [22]), there is in general a problem of mis-modeling that probably affects more or less all algorithms based on multilooking, whenever a systematic lack of triangularity is significantly present in the averaging window.

The details will depend on the specific algorithm. For example, if the temporal phase history is integrated starting from short-temporal-baseline interferograms, there could be a velocity bias induced by the following mechanism. Imagine a situation in which it rains once every now and then, and after each rainfall there is a period of progressive drying of the soil in which the SAR sensor acquires a few images. Assuming that the moisture variation produces a lack of triangularity as discussed above, the sum of the phases of "drying" interferograms will not exactly counter the phase of the "rainy" interferogram, and every rainfall will produce a phase bias in the reconstructed phase history in the millimeter or centimeter range. In this case long-term interferograms, though likely more decorrelated, might be more reliable for velocity estimation.

A possible solution to the problem could be to separate different contributions as briefly sketched in Eq. 10. An eigenvalue-eigenvector decomposition [4] might or not be an adequate tool to recover the two components, depending on their orthogonality. Once the two components have been separated, standard interferometric tools can be applied to either of them.

Another approach - only slightly different - could be to identify the parameters of a model that explain the phase mismatches and compensate the original observed coherences for the modeled coherences, so that the phases of the corrected coherences would be almost consistent, and a meaningful phase history can be extracted. In any case, there does not seem to exist an easy solution.

Propagation through variable dielectrics could be happening even without generating significant inconsistencies, for instance if a strong scattering layer is present at a few centimeter depth in the soil. In this case the interferometric processing will work fine, but the interpretation of the resulting phases as regular deformation will be wrong, since the interferometric phase of the strong scatterer will be affected by the propagation above it. The observation of closure phases over natural scatterers in general reminds us that the propagation medium could be different from air or vacuum.

\section{CONCLUSIONS}

This paper has shown with theory and examples that systematic interferometric phase mismatches between three SAR images are not unusual and can be linked to multiple scattering effects. Examples have been reported for frequencies ranging from P-band to X-band. The main candidates to explain such effects are volumetric scattering in presence of normal baselines and variations in the propagative properties of semitransparent media, chiefly moisture variations.

The presence of systematic phase inconsistencies could prevent the extraction of univocal phase histories from sample covariance matrices according to published algorithms. At the same time it has the potential to reveal additional effects which are mostly neglected in standard interferometry but are detectable with stacks. Recently started missions like ALOS-2 
and Sentinel-1, with their capability of acquiring large stacks of coherent images, will provide numerous examples of the phenomenon described in this paper.

\section{ACKNOWLEDGMENTS}

The authors thank ESA for the TropiScat campaign data (under proposal 17216) and ERS-1 data (under proposal 8049); JAXA for the PALSAR data (under proposal RA-4 PI1118); F. Rocca, P. Prats-Iraola, M. Rodriguez-Cassolà and R. Bamler for discussions. They also acknowledge the effort and valuable suggestions of the three anonymous reviewers.

\section{REFERENCES}

[1] F. De Zan, A. Parizzi, P. Prats-Iraola, and P. López-Dekker, "A SAR interferometric model for soil moisture," Geoscience and Remote Sensing, IEEE Transactions on, vol. 52, no. 1, pp. 418 - 425, Jan 2014.

[2] J. D. Monnier, "Astrophysics with closure phases," EAS Publications Series, vol. 6, pp. 213-213, Jan 2003.

[3] R. C. Jennison, "A phase sensitive interferometer technique for the measurement of the Fourier transforms of spatial brightness distributions of small angular extent," Monthly Notices of the Royal Astronomical Society, vol. 118, pp. 276-284, Mar 1958.

[4] F. De Zan, F. Rocca, and A. Rucci, "PS processing with decorrelating targets," Proc. of the Envisat Symposium 2007, pp. 1-5, July 2007.

[5] A. Monti Guarnieri and S. Tebaldini, "On the exploitation of target statistics for SAR interferometry applications," IEEE Trans. Geosci. Remote Sensing, vol. 46, pp. 3436-3443, Nov 2008.

[6] A. Ferretti, A. Fumagalli, F. Novali, C. Prati, F. Rocca, and A. Rucci, "A new algorithm for processing interferometric data-stacks: Squeesar," Geoscience and Remote Sensing, IEEE Transactions on, vol. 49, no. 9, pp. 3460-3470, Sept 2011.

[7] S. Samiei-Esfahany and R. Hanssen, "New algorithm for InSAR stack phase triangulation using integer least squares estimation," in Geoscience and Remote Sensing Symposium (IGARSS), 2013 IEEE International, July 2013, pp. 884-887.

[8] A. Ferretti, F. Novali, F. De Zan, C. Prati, and F. Rocca, "Moving from PS to slowly decorrelating targets: A prospective view," in Synthetic Aperture Radar (EUSAR), 2008 7th European Conference on. VDE, 2008, pp. 1-3.

[9] F. Rocca, "Modeling interferogram stacks," Geoscience and Remote Sensing, IEEE Transactions on, vol. 45, no. 10, pp. 3289-3299, Oct. 2007.

[10] P. Berardino, G. Fornaro, R. Lanari, and E. Sansosti, "A new algorithm for surface deformation monitoring based on small baseline differential SAR interferograms," Geoscience and Remote Sensing, IEEE Transactions on, vol. 40, no. 11, pp. 2375-2383, Nov 2002.

[11] R. Lanari, F. Casu, M. Manzo, G. Zeni, P. Berardino, M. Manunta, and A. Pepe, "An overview of the Small BAseline Subset algorithm: a DInSAR technique for surface deformation analysis," Pure and Applied Geophysics, vol. 164, no. 4, pp. 637-661, Apr 2007.

[12] M. Nolan and D. R. Fatland, "Penetration depth as a DInSAR observable and proxy for soil moisture," IEEE Trans. Geosci. Remote Sensing, vol. 41, pp. 532-537, Mar 2003.

[13] N. Mukunda and Simon R., "Quantum kinematic approach to the geometric phase," Annals of Physics, vol. 228, no. 2, pp. $205-268$, Dec 1993.

[14] S. Hensley, "Advanced radar interferometry lecture notes," in IEEE International Geoscience and Remote Sensing Symposium, 2004.

[15] S. Wollstadt, P. Lopez-Dekker, P. Prats-Iraola, F. De Zan, T. Busche, and G. Krieger, "1 and 5 day differential InSAR under crossing orbits with TerraSAR-X," in Geoscience and Remote Sensing Symposium (IGARSS), 2012 IEEE International, July 2012, pp. 1868-1871.

[16] K. Morrison, J.C. Bennett, and M. Nolan, "Using DInSAR to separate surface and subsurface features," Geoscience and Remote Sensing, IEEE Transactions on, vol. 51, no. 6, pp. 3424-3430, June 2013.

[17] J. Dall, "InSAR elevation bias caused by penetration into uniform volumes," Geoscience and Remote Sensing, IEEE Transactions on, vol. 45, no. 7, pp. 2319-2324, July 2007.

[18] R. Horn, "The DLR airborne SAR project E-SAR," in IEEE International Geoscience and Remote Sensing Symposium, May 1996, vol. 3, pp. 1624-1628.

[19] C. Albinet, P. Borderies, T. Koleck, F. Rocca, S. Tebaldini, L. Villard, T. Le Toan, A. Hamadi, and D. Ho Tong Minh, "Tropiscat: A ground based polarimetric scatterometer experiment in tropical forests," Selected Topics in Applied Earth Observations and Remote Sensing, IEEE Journal of, vol. 5, no. 3, pp. 1060-1066, June 2012.

[20] K.C. McDonald, R. Zimmermann, and J.S. Kimball, "Diurnal and spatial variation of xylem dielectric constant in Norway Spruce (Picea abies [L.] Karst.) as related to microclimate, xylem sap flow, and xylem chemistry," Geoscience and Remote Sensing, IEEE Transactions on, vol. 40, no. 9, pp. 2063-2082, Sep 2002.

[21] F. Lombardini, "Differential tomography: a new framework for sar interferometry," Geoscience and Remote Sensing, IEEE Transactions on, vol. 43, no. 1, pp. 37-44, Jan 2005.

[22] M. Schmitt and U. Stilla, "Maximum-likelihood-based approach for single-pass synthetic aperture radar tomography over urban areas," Radar, Sonar Navigation, IET, vol. 8, no. 9, pp. 1145-1153, Sept 2014. 
Francesco De Zan received his Master level degree in Telecommunication Engineering from "Politecnico di Milano" (Italy) in 2004. In 2008 he obtained a PhD degree from "Politecnico di Milano". During his PhD study he worked on extending Permanent Scatter Interferometry to decorrelating targets and contributed to ESA studies in preparation for Sentinel-1. He has been employed since 2008 at DLR (German Aerospace Center) first at the Microwaves and Radar Institute and then at the Remote Sensing Technology Institute. He has been involved

PLACE

HERE in studies for numerous future interferometric SAR missions and in calibration activities of the TanDEM-X interferometer. His research interests include algorithms and theoretical bounds for shift estimation and SAR interferometry, and interpretation of phase and coherence signatures.

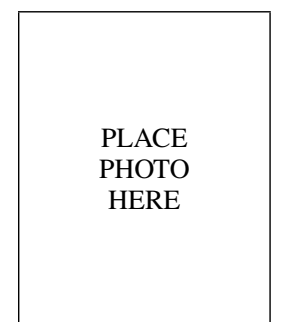

Mariantonietta Zonno was born in Bari, Italy, in 1986. She received the M. S. degree in telecommunication engineering (with honor) in 2011 from Politecnico di Bari. In March 2015 she obtained the $\mathrm{Ph} . \mathrm{D}$ degree with a thesis on synthetic aperture radar (SAR) processing (focusing and interferometric applications) from "Scuola Interpolitecnica di Dottorato" (SIPD) and Politecnico di Bari. During her $\mathrm{Ph} . \mathrm{D}$ she visited for eight months the Microwaves and Radar Institute at the German Aerospace Center (DLR), Wessling, Germany. Since November 2014 she is with the SAR Mission Group at the Microwaves and Radar Institute and her major interests are in performance analysis of future SAR missions.

\begin{tabular}{|c|}
\hline \\
PLACE \\
PHOTO \\
HERE \\
\end{tabular}

Paco López-Dekker was born in Nijmegen, The Netherlands, in 1972. He received the Ingeniero degree in telecommunication engineering from the Universitat Politècnica de Catalunya (UPC), Barcelona, Spain, in 1997, the M.S. degree in electrical and computer engineering from the University of California, Irvine, in 1998, under the Balsells Fellowships, and the Ph.D. degree from the University of Massachusetts, Amherst, in 2003, for his research on clear-air imaging radar systems to study the atmospheric boundary layer. From 1999 to 2003, he was with the Microwave Remote Sensing Laboratory, University of Massachusetts. In 2003, he was with Starlab, which is a privately held company, where he worked on the development of GNSS-R sensors. From 2004 to 2006, he was a Visiting Professor with the Telecommunications and Systems Engineering Department, Universitat Autonoma de Barcelona. In March 2006, he joined the Remote Sensing Laboratory, UPC, where he conducted research on bistatic synthetic aperture radar (SAR) under a five-year Ramon y Cajal Grant. At the university, he taught courses on signals and systems, signal processing, communications systems and radiation, and guided waves. Since November 2009, he leads the SAR Missions Group at the Microwaves and Radar Institute, German Aerospace Center, Oberpfaffenhofen, Germany. His current research is focused on the study of future SAR missions and novel mission concepts. 